\title{
Scattering of the Radial Focusing Mass-Supercritical and Energy-Subcritical Nonlinear Schrödinger Equation in 3D
}

\author{
Mujahid Abd Elmjed M-Ali ${ }^{1}$, Amadu Fullah Kamara ${ }^{2}$ \\ ${ }^{1}$ Department of Mathematics, Faculty of Education, Kassala University, Kassala, Sudan \\ ${ }^{2}$ Department of Mathematics, Faculty of Pure and Applied Sciences, Fourah Bay College, \\ University of Sierra Leone, Freetown, Sierra Leone \\ Email:mujahid@mail.ustc.edu.cn,amadu_fullah2005@yahoo.com
}

Received October 2, 2012; revised November 5, 2012; accepted November 13, 2012

\begin{abstract}
This paper studies the global behavior to 3D focusing nonlinear Schrödinger equation (NLS), the scaling index here is $\left(0<s_{c}<1\right)$, which is the mass-supercritical and energy-subcritical, and we prove under some condition the solution $u(t)$ is globally well-posed and scattered. We also show that the solution "blows-up in finite time" if the solution is not globally defined, as $t \rightarrow T$ we can provide a depiction of the behavior of the solution, where $T$ is the "blow-up time".
\end{abstract}

Keywords: NLS; Blows-Up in Finite Time; Supremum; Precompactness

\section{Introduction}

Consider the Cauchy problem for the nonlinear Schrödinger equation (NLS) in dimensions $\mathrm{d}=3$ :

$$
\left\{\begin{array}{c}
i u_{t}+\Delta u+|u|^{2} u=0 \\
u(x, 0)=u_{0}(x) \in H^{1}\left(R^{3}\right)
\end{array}\right\}
$$

where $u=u(x, t)$ is a complex-valued function in $\mathbb{R}^{3} \times \mathbb{R}$. The initial-value problem $u_{0}=u(x, 0)$ is locally well-posed in $H^{1}$.

In this paper we will study the focusing (NLS) problem, which is the mass-supercritical and energy-subcritical, where $\left(0<s_{c}<1\right)$.

The Equation (1.1) has mass $M[u](t)=M\left[u_{0}\right]$ where

$$
M[u](t)=\int|u(x, t)|^{2} \mathrm{~d} x,
$$

Energy $E[u](t)=E\left[u_{0}\right]$ where

$$
E[u](t)=\frac{1}{2} \int|\nabla u(x, t)|^{2}-\frac{1}{4} \int|u(x, t)|^{4} \mathrm{~d} x,
$$

and Momentum $P[u](t)=P\left[u_{0}\right]$ where

$$
P[u](t)=\operatorname{Im} \int \bar{u}(x, t) \nabla u(x, t) \mathrm{d} x .
$$

If $\left\|x u_{0}\right\|_{L^{2}}<\infty$, then $u$ satisfies

$$
\partial_{t}^{2} \int|x|^{2}|u(x, t)|^{2} \mathrm{~d} x=24 E[u]-4\|\nabla u(t)\|_{L_{x}^{2}}^{2}
$$

Equation (1.2) is said to be the Virial identity.
The Equation (1.1) has the scaling:

$$
u_{\lambda}(x, t)=\lambda u\left(\lambda x, \lambda^{2} t\right)
$$

and also this scaling is a solution if $u(x, t)$ is a solution.

Moreover, $u_{0}$ is a solution that is globally defined by $u$, if it is globally defined $(T=+\infty)$, and it does scatter (See $[1,2])$. We say the solution "blows-up in finite time". If the solution is not globally defined, as $t \rightarrow T$, we can provide a depiction of the behavior of the solution, where $T$ is the "blow-up time". It follows from the $H^{1}$ local theory optimized by scaling, that if blow-up in finite-time $T$ $>0$ happens, (see [3] or [4]), then there is a lower-bound on the "blow-up rate":

$$
\|\nabla u(t)\|_{L_{x}^{2}} \geq \frac{c}{(T-t)^{\frac{1}{4}}}
$$

for some constant $c$. Thus, to prove global presence, it suffices to prove a global axiomatic bound on $\|\nabla u(t)\|_{L^{2}}$. From the Strichartz estimates, there is a constant $c_{S T}>0$ such that if $\left\|u_{0}\right\|_{H^{2}} \frac{1}{2}<c_{S T}$, then the solution $u$ is globally defined and scattered.

Note that the quantities $\left\|u_{0}\right\|_{L^{2}} \nabla u_{0_{L^{2}}}$ and $M\left[u_{0}\right] E\left[u_{0}\right]$ are also scale-invariant (See also [5]).

Let $u(x, t)=\mathrm{e}^{i t} \psi(x)$ then $u$ solves (1.1) as long as $\psi$ solves the nonlinear elliptic equation

$$
-\psi+\Delta \psi+|\psi|^{2} \psi=0
$$


Equation (1.4) has an infinite number of solutions in $H^{1}\left(\mathbb{R}^{3}\right)$. The solution of minimal mass is denoted by $\psi(x)$ and for the properties of $\psi$ see $[3,5,6]$.

Under the condition $M[u] E[u]<M[\psi] E[\psi]$, solutions to (1.1) globally exist if $u_{0}$ satisfies;

$$
\left\|u_{0}\right\|_{L^{2}}\left\|\nabla u_{0}\right\|_{L^{2}}<\|\psi\|_{L^{2}}\|\nabla \psi\|_{L^{2}},
$$

and there exist $\phi_{ \pm} \in H^{1}$ such that

$$
\lim _{t \rightarrow \pm \infty}\left\|u(t)-\mathrm{e}^{i t \nabla} \phi_{ \pm}\right\|_{H^{1}}=0 .
$$

Theorem 1.1. Let $u_{0} \in H^{1}$, and let $u$ be the corresponding solution to (1.1) in $H^{1}$. Suppose

$$
M[u] E[u]<M[\psi] E[\psi]
$$

If $\left\|u_{0}\right\|_{L^{2}}\left\|\nabla u_{0}\right\|_{L^{2}}<\|\psi\|_{L^{2}}\|\nabla \psi\|_{L^{2}}$ then $u$ scatters in $H^{1}$.

The argument of [6] in the radial case followed a strategy introduced by [7] for proving global well-posedness and scattering for the focusing energy-critical NLS. The beginning used a contradiction to the argument: suppose the sill for scattering is strictly below that claimed. This uniform localization enabled the use of a local Virial identity to be established, with the support of the sharp Gagliardo-Nirenberg inequality, an accurately positive lower bound on the convexity (in time) of the local mass of $u_{c}$ Mass conservation is then violated at enough large time.

We show in this paper, that the above program carries over to the non-radial setting with the extension of two key components.

Theorem 1.2. Suppose the radial $H^{1}$ solution $u$ to (1.1) blows-up at time $T<\infty$. Then either there is a non-absolute $c_{1} \gg 1$ constant such that, as $t \rightarrow T$

$$
\int_{|x| \leq c_{1}^{2} \| \nabla u(t)||_{L^{2}}^{-2}}|u(x, t)|^{3} \mathrm{~d} x \geq c_{1}^{-1},
$$

or there exists a sequence of times $t_{n} \rightarrow T$ such that for an absolute constant $c_{2}$

$$
\int_{\frac{3}{3}|x| \leq\left. c_{2}\left|u_{0}\right|\right|_{L^{2}} ^{2}\|\nabla u(t) \mid\|_{L^{2}}^{\frac{-1}{2}}}\left|u\left(x, t_{n}\right)\right|^{3} \mathrm{~d} x \rightarrow \infty
$$

From (1.3), we have that the concentration in (1.7) satisfies $\|\nabla u(t)\|_{L^{2}}^{-2} \leq c(T-t)^{\frac{1}{2}}$, and the concentration in (1.8) satisfies $\|\nabla u(t)\|_{L^{2}}^{\frac{-1}{2}} \leq c(T-t)^{\frac{1}{8}}$ (For more additional information see [8-10]).

\section{Notation}

Let $\mathrm{e}^{i t \Delta} f$ be the free Schrödinger propagator, and let $u_{t}+\nabla u=0$, with $u(0, x)=f(x)$ be linear equation, a solution in physical space, is given by:

$$
\mathrm{e}^{i t \Delta} f(x)=\frac{1}{(4 \pi i t)^{\frac{d}{2}}} \int_{R^{d}} \mathrm{e}^{\frac{i|x-y|^{2}}{4 t}} f(y) \mathrm{d} y,
$$

and in frequency space

$$
\mathrm{e}^{\hat{i t \Delta}} f(\xi)=\mathrm{e}^{-4 \pi^{2} i t|\xi|^{2}} \hat{f}(\xi)
$$

In particular, they save the Farewell homogeneous Sobolev norms and obey the dispersive inequality

$$
\left\|\mathrm{e}^{i t \Delta} f\right\|_{L_{x}^{\infty}\left(R^{d}\right)} \lesssim|t|^{-\frac{d}{2}} f_{L_{x}^{1}}
$$

For all times $t \neq 0$.

Let $\phi(x) \in C_{c}^{\infty}\left(\mathbb{R}^{3}\right)$ be a radial function, so that, $\phi(x)=1$ for $|x| \leq 1$ and $\phi(x)=0$ for $|x| \geq 2$, Define the inner and outer spatial localizations of $u(x, t)$ at radius $R(t)>0$ as

$$
\begin{gathered}
u_{1}(x, t)=\phi(x / R(t)) u(x, t), \\
u_{2}(x, t)=(1-\phi(x / R(t))) u(x, t)
\end{gathered}
$$

Let $\chi(x) \in C_{c}^{\infty}\left(\mathbb{R}^{3}\right)$ be a radial function so that, $\chi(x)=1$ for $|x| \leq \frac{1}{8 \pi}$ and $\chi(x)=0$ for $|x| \geq \frac{1}{2 \pi}$ then $\hat{\chi}(0)=1$, and define the inner and outer indecision localizations at radius $\rho(t)$ of $u_{1}$ as

$$
\hat{u}_{1 L}(\xi, t)=\hat{\chi}(\xi / \rho(t)) \hat{u}_{1}(\xi, t),
$$

and

$$
\hat{u}_{1 H}(\xi, t)=(1-\hat{\chi}(\xi / \rho(t))) \hat{u}_{1}(\xi, t),
$$

(the $\frac{1}{8 \pi}$ and $\frac{1}{2 \pi}$ radii are chosen to be consistent with the assumption $\hat{\chi}(0)=1$, since $\hat{\chi}(0)=\int_{R^{3}} \chi(x) \mathrm{d} x$. In reality, this is for suitability only; the argument is easily proper to the case where $\hat{\chi}(0)$ is any number $\neq 0$ ). We note that the indecision localization of $u_{1}=u_{1 L}+u_{1 H}$ is inaccurate, though decisively we have;

$$
|1-\hat{\chi}(\xi)| \leq c \min (|\xi|, 1)
$$

\section{Proof of Theorem 1.2}

In this section we discuss a proof of Theorem (1.2).

Proposition 2.1. Let $u$ be an $H^{1}$ radial solution to (1.1) that blows-up in finite $T>0$. Let

$$
R(t)=c_{1}\left\|u_{0}\right\|_{L^{2}}^{\frac{3}{2}}\|\nabla u(t)\|_{L^{2}}^{-\frac{1}{2}}
$$

and $\rho(t)=c_{2}\|\nabla u(t)\|_{L_{x}^{2}}^{2}$, (Where $c_{1}$ and $c_{2}$ are absolute constants), and $u=u_{1 L}+u_{1 H}+u_{2}$ as characterized in the paragraph above. 
1) There exists an absolute constant $c>0$ such that

$$
\left\|u_{1 L}(t)\right\|_{L_{x}^{3}} \geq c \text { as } t \rightarrow T .
$$

2) Let us assume that there exists a constant $c^{*}$ such that $\left\|u_{1}(t)\right\|_{L^{3}} \leq c^{*}$. Then

$$
\left\|u_{1}(t)\right\|_{L_{x}^{3} \mid\left(x-x_{0}(t) \mid \leq \rho(t)^{-1}\right)} \geq \frac{c}{\left(c^{*}\right)^{3}} \text { as } t \rightarrow T
$$

for some absolute constant $c>0$, where $x_{0}(t)$ is a stance function such that

$$
\left|x_{0}(t)\right| / \rho(t)^{-1} \leq c \cdot\left(c^{*}\right)^{6} .
$$

We recall, an "exterior" estimate, usable to radially symmetric functions only, originally due to [11]:

$$
\|v\|_{L_{\{|x|>R\}}^{4}}^{4} \leq \frac{c}{R^{2}} v_{L_{\{|x|>R\}}^{2}}^{3} \nabla v_{L_{\{|x|>R\}}^{2}}
$$

where $c$ is independent of $R>0$. We recall the generally usable symmetric functions and for any function $v$,

$$
\|v\|_{L_{\left(R^{3}\right)}^{4}}^{4} \leq c\|v\|_{L_{\left(R^{3}\right)}^{3}}^{2}\|\nabla v\|_{L_{R^{3}}^{2}}^{2} .
$$

(2.3), (2.4) are Gagliardo-Nirenberg estimates for functions on $\mathbb{R}^{3}$.

Proof of Prop 2.1: Since by (1.3), $\|\nabla u(t)\|_{L_{x}^{2}} \rightarrow+\infty$ as $t \rightarrow T$, by energy conservation, we have $u(t)_{L_{x}^{4}}^{4} / \nabla u(t)_{L_{x}^{2}}^{2} \rightarrow 2$. Thus, for $t$ to be large enough to close to $T$

$$
\|\nabla u\|_{L_{x}^{2}}^{2} \leq u_{L_{x}^{4}}^{4} \leq\left\|u_{1 L}\right\|_{L_{x}^{4}}^{4}+\left\|u_{1 H}\right\|_{L_{x}^{4}}^{4}+\left\|u_{2}\right\|_{L_{x}^{4}}^{4} .
$$

By (2.3), the selection of $R(t)$ and mass conservation;

$$
\left\|u_{2}\right\|_{L_{x}^{4}}^{4} \leq \frac{c}{R^{2}}\left\|u_{0}\right\|_{L_{x}^{2}}^{3}\|\nabla u\|_{L_{x}^{2}} \leq \frac{1}{4}\|\nabla u\|_{L_{x}^{2}}^{2}
$$

where $c_{1}$ in the definition of $R(t)$ has been selected to obtain the factor $\frac{1}{4}$ here. By Sobolev embedding, (1.10), and the selected $\rho(t)$

$$
\begin{aligned}
\left\|u_{1 H}\right\|_{L_{x}^{4}}^{4} & \leq c\left\|u_{1 H}\right\|_{\dot{H}_{x}^{\frac{3}{4}}}^{4}=c\left\|\left.\xi\right|^{\frac{3}{4}}(1-\hat{\chi}(\xi / \rho)) \hat{u}_{1}(\xi)\right\|_{L_{\xi}^{2}}^{4} \\
& \leq c \rho^{-1}\left\|\xi \mid \hat{u}_{1}(\xi)\right\|_{L_{\xi}^{2}}^{4} \leq c \rho^{-1}\left\|\nabla u_{1}\right\|_{L_{x}^{2}}^{4} \\
& \leq c \rho^{-1}\|\nabla u\|_{L_{x}^{2}}^{4} \leq \frac{1}{4} \nabla u_{L_{x}^{2}}^{2}
\end{aligned}
$$

where $c_{2}$ in the definition of $\rho(t)$ has been selected to obtain the factor $\frac{1}{4}$ here. Bring together (2.5), (2.6), and (2.7), to obtain

$$
\|\nabla u\|_{L_{x}^{2}}^{2} \leq c\left\|u_{1 L}\right\|_{L_{x}^{4}}^{4}
$$

By (2.8) and (2.4), we obtain (2.1), completing the proof of part (1) of the proposition.

To prove part (2), we assume $u_{1}(t)_{L^{3}} \leq c^{*}$, by (2.8)

$$
\begin{aligned}
\|\nabla u\|_{L^{2}}^{2} & \leq c\left\|u_{1 L}\right\|_{L_{x}^{3}}^{3}\left\|u_{1 L}\right\|_{L_{x}^{\infty}} \leq c \cdot\left(c^{*}\right)^{3}\left\|u_{1 L}\right\|_{L_{x}^{\infty}} \\
& \leq c \cdot\left(c^{*}\right)^{3} \sup _{x \in R^{3}} \int \rho^{3} \chi \rho(x-y) u_{1}(y) \mathrm{d} y \mid .
\end{aligned}
$$

There exists $x_{0}=x_{0}(t) \in \mathbb{R}^{3}$ for which at least $\frac{1}{2}$ of this supremum is attained. Thus,

$$
\begin{aligned}
\|\nabla u\|_{L^{2}}^{2} \leq & c \cdot\left(c^{*}\right)^{3}\left|\int \rho^{3} \chi \rho\left(x_{0}-y\right) u_{1}(y) \mathrm{d} y\right| \\
& \cdot\left|\int \rho^{3} \chi \rho\left(x_{0}-y\right) u_{1}(y) \mathrm{d} y\right| \\
\leq & c \cdot\left(c^{*}\right)^{3} \rho^{3} \int_{\left|x_{0}(t)-y\right| \leq \rho^{-1}}\left|u_{1}(y)\right| \mathrm{d} y \\
\leq & c \cdot\left(c^{*}\right)^{3} \rho\left(\int_{\left|x_{0}(t)-y\right| \leq \rho^{-1}}\left|u_{1}(y)\right|^{3} \mathrm{~d} y\right)^{\frac{1}{3}}
\end{aligned}
$$

where we used Hölder's inequality in the last step. By the selected $\rho$, we obtain (2.2). To complete the proof, it keeps to obtain the remind control on $x_{0}(t)$ which will be a consequence of the radial supposition and the supposed bound $\left\|u_{1}(t)\right\|_{L^{3}} \leq c^{*}$.

Assume $\frac{\left|x_{0}\left(t_{n}\right)\right|}{\rho\left(t_{n}\right)^{-1}} \gg\left(c^{*}\right)^{6}$ along a sequence of times $t_{n} \rightarrow T$. Assume the spherical annulus;

$$
A=\left\{x \in \mathbb{R}^{3}:\left|x_{0}\right|-\rho^{-1} \leq|x| \leq\left|x_{0}\right|+\rho^{-1}\right\} .
$$

And inside $A$ place $\sim \frac{4 \pi\left|x_{0}\right|^{2}}{\pi\left(\rho^{-1}\right)^{2}}$ disjoint balls, at radius $x_{0}$, both the radius $\rho^{-1}$, centered on the sphere. By the radiality supposition, on all ball $B$, we have $\left\|u_{1}\right\|_{L_{B}^{3}} \geq \frac{c}{\left(c^{*}\right)^{3}}$, and hence on the annulus $A$,

$$
\left\|u_{1}\right\|_{L_{A}^{3}}^{3} \geq \frac{c}{\left(c^{*}\right)^{9}} \frac{\left|x_{0}\right|^{2}}{\left(\rho^{-1}\right)^{2}} \gg\left(c^{*}\right)^{3} .
$$

which contradicts the assumption $\left\|u_{1}\right\|_{L^{3}} \leq c^{*}$.

We now point out how to obtain Theorem 1.2 as a consequence.

Proof of Theorem 1.2. By part (1) of Prop. 2.1 and the standard convolution inequality:

$$
c \leq\left\|u_{1 L}\right\|_{L_{x}^{3}}=\left\|\rho^{3} \chi(\rho \cdot) * u_{1}\right\|_{L_{x}^{3}} \leq\left\|u_{1}\right\|_{L^{3}} .
$$


If $\left\|u_{1}(t)\right\|_{L^{3}}$ is not bounded, then there exists a sequence of times $t_{n} \rightarrow T$ such that $\left\|u_{1}\left(t_{n}\right)\right\|_{L^{3}} \rightarrow \infty$. Since $\left\|u\left(t_{n}\right)\right\|_{L^{3}(|x| \leq 2 R)} \geq u_{1}\left(t_{n}\right)_{L^{3}}$, we have (1.8) in Theorem 1.2; on the other hand, if $\left\|u_{1}(t)\right\|_{L^{3}} \leq c^{*}$, for some $c^{*}$, as $t \rightarrow T$, we have (2.2) of Prop. 2.1. Since $\left|x_{0}(t)\right| \leq c\left(c^{*}\right)^{6} \rho(t)^{-1}$, we have

$$
\frac{c}{\left(c^{*}\right)^{3}} \leq\left\|u_{1}(t)\right\|_{L^{3}\left(\left|x-x_{0}(t)\right| \leq \rho(t)^{-1}\right)} \leq\left\|u_{1}\left(t_{n}\right)\right\|_{L^{3}\left(|x| \leq c\left(c^{*}\right)^{6} \rho(t)^{-1}\right)}
$$

which gives (1.7) in Theorem 1.2.

\section{Strichartz Estimates}

In this section we show local theory and Strichartz estimates.

\section{Strichartz Type Estimates}

We say the pair $(q, r)$ is $\dot{H}^{s}$ Strichartz admissible if $\frac{2}{q}+\frac{d}{r}=\frac{d}{2}-s$, with $2 \leq q, r \leq \infty$ and $(q, r, d) \neq(2, \infty, 2)$.

And the pair $(q, r)$ is $\frac{d}{2}$-passable if $1 \leq q, r \leq \infty$, $\frac{1}{q}<d\left(\frac{1}{2}-\frac{1}{r}\right)$ or $(q, r)=(\infty, 2)$.

As habitual we denote by $q^{\prime}, r^{\prime}$ the Hölder conjugates of $q$ and $r$ consecutive (i.e. $\frac{1}{r}+\frac{1}{r^{\prime}}=1$ ).

Let

$$
\|u\|_{S\left(L^{2}\right)}=\sup _{(q, r) L^{2} \text { admissible } 2 \leq r \leq 6,2 \leq q \leq \infty}\|u\|_{L_{L}^{q} L_{x}^{r}} .
$$

We consider dual Strichartz norms. Let

$$
u_{S^{\prime}\left(L^{2}\right)}=\inf _{(q, r) L^{2} \text { admissible } 2 \leq q \leq \infty, 2 \leq r \leq 6,}\|u\|_{L_{t}^{q^{\prime}} L_{x}^{r^{\prime}}} .
$$

where $\left(q^{\prime}, r^{\prime}\right)$ is the Hölder dual to $(q, r)$. Also define

$$
\|u\|_{S^{\prime}}\left(\dot{H}^{-\frac{1}{2}}\right)=\sup _{(q, r) \dot{H}^{-\frac{1}{2}}}{\operatorname{sadmissible~} \frac{4^{+}}{3} \leq q \leq 6^{-}, 3^{+} \leq r \leq 6^{-}}_{L_{t}^{q^{\prime} L_{x}^{\prime}}}
$$

The Strichartz estimates are:

$$
\left\|\mathrm{e}^{i t \Delta} \phi\right\|_{S\left(L^{2}\right)} \leq c\|\phi\|_{L^{2}}
$$

and

$$
\left\|\int_{0}^{t} \mathrm{e}^{i\left(t-t^{\prime}\right) \Delta} f\left(\cdot, t^{\prime}\right) \mathrm{d} t^{\prime}\right\|_{S\left(L^{2}\right)} \leq c\|f\|_{S^{\prime}\left(L^{2}\right)} .
$$

By bring together Sobolev embedding with the Strichartz estimates, we obtain

$$
\left\|\mathrm{e}^{i t \Delta} \phi\right\|_{S}\left(\dot{H}^{\frac{1}{2}}\right) \leq c\|\phi\|_{\dot{H}^{\frac{1}{2}}}
$$

and

$$
\left.\left\|\int_{0}^{t} \mathrm{e}^{i\left(t-t^{\prime}\right) \Delta} f\left(\cdot, t^{\prime}\right) \mathrm{d} t^{\prime}\right\|_{S} \dot{H}^{\frac{1}{2}}\right) \leq c\left\|D^{\frac{1}{2}} f\right\|_{S^{\prime}\left(L^{2}\right)} .
$$

We must also need the Kato inhomogeneous Strichartz estimate [12].

$$
\left\|\int_{0}^{t} \mathrm{e}^{i\left(t-t^{\prime}\right) \Delta} f\left(\cdot, t^{\prime}\right) \mathrm{d} t^{\prime}\right\|_{S\left(\dot{H}^{\frac{1}{2}}\right)} \leq c\|f\|_{S^{\prime}}\left(\dot{H}^{-\frac{1}{2}}\right) .
$$

To point out a restriction to a time subinterval $\subset(-\infty,+\infty)$, we will write $S\left(\dot{H}^{s} ; I\right)$ or $S^{\prime}\left(\dot{H}^{s} ; I\right)$.

Proposition 3.1 Assume $\left\|u_{0}\right\|_{S}\left(\dot{H}^{\frac{1}{2}}\right) \leq M$. There is $\delta_{s d}=\delta_{s d}(M)>0$ such that if $\left\|\mathrm{e}^{i t \Delta} u_{0}\right\|_{S\left(\dot{H}^{\frac{1}{2}}\right)} \leq \delta_{\text {sd }}$, then $u$ solving (1.1) is global (in $\dot{H}^{\frac{1}{2}}$ ) and

$$
\begin{gathered}
\|u\|_{S}\left(\dot{H}^{\frac{1}{2}}\right) \leq 2\left\|\mathrm{e}^{i t \Delta} u_{0}\right\|_{S}\left(\dot{H}^{\frac{1}{2}}\right), \\
\left\|D^{\frac{1}{2}} u\right\|_{S\left(\dot{L}^{2}\right)} \leq 2 c\left\|u_{0}\right\|_{\dot{H}^{\frac{1}{2}}} .
\end{gathered}
$$

(Observe that, by the Strichartz estimates, the assumptions are satisfied if $\left.\left\|u_{0}\right\|_{\dot{H}^{2}} \leq c \delta_{s d}\right)$.

Proof. Define

$$
\Psi_{u_{0}}(v)=\mathrm{e}^{i t \Delta} u_{0}+i \int_{0}^{t} \mathrm{e}^{i\left(t-t^{\prime}\right) \Delta}|v|^{2} v\left(t^{\prime}\right) \mathrm{d} t^{\prime} .
$$

Applying the Strichartz estimates, we obtained

$$
\left\|D^{\frac{1}{2}} \Psi_{u_{0}}(v)\right\|_{S\left(\dot{L}^{2}\right)} \leq c\left\|u_{0}\right\|_{\dot{H}^{\frac{1}{2}}}+c\left\|D^{\frac{1}{2}}\left(|v|^{2} v\right)\right\|_{L_{t}^{\frac{5}{2}} \frac{10}{9}}
$$

and

$$
\left\|\Psi_{u_{0}}(v)\right\|_{S}\left(\dot{H}^{\frac{1}{2}}\right) \leq c\left\|\mathrm{e}^{i t \Delta} u_{0}\right\|_{S}\left(\dot{H}^{\frac{1}{2}}\right)+c\left\|D^{\frac{1}{2}}\left(|v|^{2} v\right)\right\|_{L_{t}^{\frac{5}{2}} \frac{10}{9}}
$$

We apply the Hölder inequalities and fractional Leibnitz [13] to get

$$
\left.\left\|D^{\frac{1}{2}}\left(|v|^{2} v\right)\right\|_{\frac{5}{L_{t}^{2}} \frac{10}{9}} \leq\|v\|_{L_{t}^{5} L_{x}^{5}}^{2}\left\|D^{\frac{1}{2}} v\right\|_{L^{\infty} L^{2}} \leq\|v\|_{S}^{2} \dot{H}^{\frac{1}{2}}\right)\left\|D^{\frac{1}{2}} v\right\|_{S\left(\dot{L}^{2}\right)}
$$

Let

$$
\delta_{s d} \leq \min \left(\frac{1}{\sqrt{24} c}, \frac{1}{24 c M}\right) .
$$

Then $\Psi_{u_{0}}: N \rightarrow N$, where 


$$
N=\left\{v \mid\|v\|_{S}\left(\dot{H}^{\frac{1}{2}}\right) \leq\left\|2 \mathrm{e}^{i t \Delta} u_{0}\right\|_{S}\left(\dot{H}^{\frac{1}{2}}\right),\left\|D^{\frac{1}{2}} v\right\|_{S\left(\dot{L}^{2}\right)} \leq 2 c\left\|u_{0}\right\|_{\dot{H}^{\frac{1}{2}}}\right\}
$$

and $\Psi_{u_{0}}$ is a contraction on $N$.

Proposition 3.2. If $u_{0} \in H^{1}, u(t)$ is global with globally finite $\dot{H}^{\frac{1}{2}}$ Strichartz norm $\|u\|_{S}\left(\dot{H}^{\frac{1}{2}}\right)<+\infty$ and a uniformly bounded $H^{1}$ norm $\sup _{t \in[0,+\infty)}\|u(t)\|_{H^{1}} \leq N$, then $u(t)$ scatters in $H^{1}$ as $t \rightarrow+\infty$.

Meaning that there exist $\phi^{+} \in H^{1}$ such that

$$
\lim _{t \rightarrow+\infty}\left\|u(t)-\mathrm{e}^{i t \Delta} \phi^{+}\right\|_{H^{1}}=0 .
$$

Proof. Since $u(t)$ resolves the integral equation

$$
u(t)=\mathrm{e}^{i t \Delta} u_{0}+i \int_{0}^{t} \mathrm{e}^{i\left(t-t^{\prime}\right) \Delta}\left(|u|^{2} u\right)\left(t^{\prime}\right) \mathrm{d} t^{\prime},
$$

we have

$$
u(t)-\mathrm{e}^{i t \Delta} \phi^{+}=-i \int_{t}^{+\infty} \mathrm{e}^{i\left(t-t^{\prime}\right) \Delta}\left(|u|^{2} u\right)\left(t^{\prime}\right) \mathrm{d} t^{\prime}
$$

where

$$
\phi^{+}=u_{0}+i \int_{0}^{+\infty} \mathrm{e}^{-i t^{\prime} \Delta}\left(|u|^{2} u\right)\left(t^{\prime}\right) \mathrm{d} t^{\prime} .
$$

Apply the Strichartz estimates to (3.3), to get

$$
\begin{aligned}
& \left\|u(t)-\mathrm{e}^{i t \Delta} \phi^{+}\right\|_{H^{1}} \\
& \leq c\left\||u|^{2}(1+|\nabla|) u\right\|_{\left.L_{[l,+\infty}^{\frac{5}{2}}\right)_{x}^{\frac{10}{9}}} \\
& \leq c\|u\|_{\left.L_{[t,+\infty}^{5}\right)^{5}}^{2} L_{x}^{5}\|u\|_{L_{t}^{\infty} H_{x}^{1}} \leq c N\|u\|_{\left.L_{[t,+\infty}^{5}\right)^{2}}^{L_{x}^{5}}
\end{aligned}
$$

As $t \rightarrow \infty$ above inequality get the claim.

\section{Some Lemma}

\subsection{Here We Discuss the Precompactness of the Flow Implies Regular Localization}

Let $u$ be a solution to (1.1) such that

$$
K=\{u(\cdot-\eta(t), t) \mid t \in[0,+\infty)\}
$$

is precompact in $H^{1}$. Then for each $\varepsilon>0$ there exist $R>0$ so that $\pi$ for all $0 \leq t<+\infty$.

We proof (4.2) by contradiction, there exists $\varepsilon>0$ and a sequence of times $t_{n}$ and by changing the variables,

$$
\begin{aligned}
& \int_{|\eta|>n}\left|\nabla u\left(\eta-\eta\left(t_{n}\right), t_{n}\right)\right|^{2}+\left|u\left(\eta-\eta\left(t_{n}\right), t_{n}\right)\right|^{2} \\
& +\left|u\left(\eta-\eta\left(t_{n}\right), t_{n}\right)\right|^{4} \varepsilon \geq \varepsilon
\end{aligned}
$$

Since $K$ is precompact, there exists $\varphi \in H^{1}$, such that $u\left(\cdot-\eta\left(t_{n}\right), t_{n}\right) \rightarrow \varphi$ in $H^{1}$, by (4.3),

$$
\forall R>0, \int_{|\eta|>R}|\nabla \varphi(\eta)|^{2}+|\varphi(\eta)|^{2}+|\varphi(\eta)|^{4} \mathrm{~d} \eta \geq \varepsilon .
$$

Which is a contradiction with the fact that $\varphi \in H^{1}$. The proof is complete.

Lemma 4.1. Let $u$ be a solution of (1.1) defined on $[0,+\infty)$, such that $P[u]=0$ and $K$ such as in (4.1) is precompact in $H^{1}$, for some continuous function $\eta(\cdot)$ then;

$$
\frac{\eta(t)}{t} \rightarrow 0 \text { as } t \rightarrow+\infty
$$

Proof. Suppose that (4.4) does not hold. Then there exists a sequence $t_{n} \rightarrow+\infty$, such that $\frac{\eta\left(t_{n}\right)}{t_{n}} \geq \varepsilon_{0}$ for some $\varepsilon_{0}>0$. Retaining generality, we assume $\eta(0)=0$. For $R>0$, let

$$
t_{0}(R)=\inf \{t \geq 0:|\eta(t)| \geq R\}
$$

i.e. $t_{0}(R)$ is the first time when $\eta(t)$ arrives at the boundary of the ball of radius $R$. By continuity of $\eta(t)$, the value $t_{0}(R)$ is well-defined. Furthermore, the following hold:

1) $t_{0}(R)>0$;

2) $|\eta(t)|<R$, for $0 \leq t \leq t_{0}(R)$;

3) $\left|\eta\left(t_{0}(R)\right)\right|=R$.

Let $R_{n}=\left|\eta\left(t_{n}\right)\right|$ and $\tilde{t}_{n}=t_{0}\left(R_{n}\right)$. We note that $t_{n} \geq \tilde{t}_{n}$, which combined with $\frac{\left|\eta\left(t_{n}\right)\right|}{t_{n}} \geq \varepsilon_{0}$, gives $\frac{R_{n}}{\tilde{t}_{n}} \geq \varepsilon_{0}$. Since $t_{n} \rightarrow+\infty$ and $\frac{\left|\eta\left(t_{n}\right)\right|}{t_{n}} \geq \varepsilon_{0}$, we have $R_{n}=\left|\eta\left(t_{n}\right)\right| \rightarrow+\infty$. Thus $\tilde{t}_{n}=t_{0}\left(R_{n}\right) \rightarrow+\infty$. We can disregard $t_{n}$. We will concentrate our work on the time interval $\left[0, \tilde{t}_{n}\right]$, and we will use in the proof:

1) for $0 \leq t \leq \tilde{t}_{n}$ we have $|\eta(t)|<R_{n}$;

2) $\left|\eta\left(\tilde{t}_{n}\right)\right|=R_{n}$;

3) $\frac{R_{n}}{t_{n}} \geq \varepsilon_{0}$ and $\tilde{t}_{n} \rightarrow+\infty$.

By the precompactness of $K$ and (4.2) it follows that for any $\varepsilon>0$, there exists $R_{0}(\varepsilon) \geq 0$, such that for any $t \geq 0$

$$
\int_{|\eta+\eta(t)| \geq R_{0}(\varepsilon)}\left(|u|^{2}+|\nabla u|^{2}\right) \mathrm{d} \eta \leq \varepsilon .
$$

We will select $\varepsilon$ later; for $\eta \in \mathbb{R}$ let $\gamma(\eta) \in C_{0}^{\infty}(\mathbb{R})$ 
be such that $\gamma(\eta)=\eta$ for $-1 \leq \eta \leq 1, \gamma(\eta)=0$ for $|\eta| \geq 2^{\frac{1}{3}},|\gamma(\eta)| \leq|\eta|, \quad \gamma_{\infty}^{\prime} \leq 4$ and $\gamma_{\infty} \leq 2$ for $\eta=\left(\eta_{1}, \eta_{2}, \eta_{3}\right) \in \mathbb{R}^{3}$. Let $\phi(\eta)=\left(\gamma\left(\eta_{1}\right), \gamma\left(\eta_{2}\right), \gamma\left(\eta_{3}\right)\right)$. Then $\phi(\eta)=\eta$ for $|\eta| \leq 1$ and $\|\phi\|_{\infty} \leq 2$. For $R>0$, set $\phi_{R}(\eta)=R \phi(\eta / R)$. Let $z_{R}: \mathbb{R} \rightarrow \mathbb{R}^{3}$ be the truncation center of mass given by

$$
z_{R}(t)=\int \phi_{R}(\eta)|u(\eta, t)|^{2} \mathrm{~d} \eta .
$$

Then $z_{R}^{\prime}(t)=\left(\left[z_{R}^{\prime}(t)\right]_{1},\left[z_{R}^{\prime}(t)\right]_{2},\left[z_{R}^{\prime}(t)\right]_{3}\right)$, where

$$
\left[z_{R}^{\prime}(t)\right]_{j}=2 \operatorname{Im} \int \gamma^{\prime}\left(\eta_{j} / R\right) \partial_{j} u \bar{u} \mathrm{~d} \eta .
$$

Observe that $\gamma^{\prime}\left(\frac{\eta_{j}}{R}\right)=1$ for $\left|\eta_{j}\right| \leq 1$. By the zero momentum property

$$
\operatorname{Im} \int_{\left|\eta_{j}\right| \leq R} \partial_{j} u \bar{u}=-\operatorname{Im} \int_{\left|\eta_{j}\right|>R} \partial_{j} u \bar{u} .
$$

Thus,

$$
\begin{aligned}
{\left[z_{R}^{\prime}(t)\right]_{j}=} & -2 \operatorname{Im} \int_{\left|\eta_{j}\right| \geq R} \partial_{j} u \bar{u} \mathrm{~d} \eta \\
& +2 \operatorname{Im} \int_{\left|\eta_{j}\right| \geq R} \gamma^{\prime}\left(\eta_{j} / R\right) \partial_{j} u \bar{u} \mathrm{~d} \eta .
\end{aligned}
$$

By Cauchy-Schwarz, we obtain;

$$
\left|z_{R}^{\prime}(t)\right| \leq 5 \int_{|\eta| \geq R}\left(|u|^{2}+|\nabla u|^{2}\right) .
$$

Set $\tilde{R}_{n}=R_{n}+R_{0}(\varepsilon)$. Observe that for $0 \leq t \leq \tilde{t}_{n}$ and $|\eta| \geq \tilde{R}_{n}$, we have $|\eta+\eta(t)| \geq \tilde{R}_{n}-R_{n}=R_{0}(\varepsilon)$, and thus (4.6), (4.5) give

$$
\left|z_{\tilde{R}_{n}}^{\prime}(t)\right| \leq 5 \varepsilon .
$$

We now obtain an upper bound for $z_{\tilde{R}_{n}}^{\prime}(0)$ and a lower bound for $z_{\tilde{R}_{n}}^{\prime}(t)$

$$
\begin{aligned}
z_{\tilde{R}_{n}}^{\prime}(0)= & \int_{|\eta|<R_{0}(\varepsilon)} \phi_{\tilde{R}_{n}}(\eta)\left|u_{0}\right|^{2} \mathrm{~d} \eta \\
& +\int_{|\eta+\eta(0)| \geq R_{0}(\varepsilon)} \phi_{\tilde{R}_{n}}(\eta)\left|u_{0}\right|^{2} \mathrm{~d} \eta
\end{aligned}
$$

Hence, by (4.5) we have

$$
\left|z_{\tilde{R}_{n}}^{\prime}(0)\right| \leq R_{0}(\varepsilon) M[u]+2 \tilde{R}_{n} \varepsilon .
$$

For $0 \leq t \leq \tilde{t}_{n}$, we divide $z_{\tilde{R}_{n}}^{\prime}(t)$ as

$$
\begin{aligned}
z_{\tilde{R}_{n}}^{\prime}(t)= & \int_{|\eta+\eta(t)| \geq R_{0}(\varepsilon)} \phi_{\tilde{R}_{n}}(\eta)\left|u_{0}\right|^{2} \mathrm{~d} \eta \\
& +\int_{|\eta+\eta(t)| \geq R_{0}(\varepsilon)} \phi_{\tilde{R}_{n}}(\eta)\left|u_{0}\right|^{2} \mathrm{~d} \eta \\
= & I+I I
\end{aligned}
$$

To deduce the expression for $I$, we observed that $\left|\phi_{\tilde{R}_{n}}(\eta)\right| \leq 2 \tilde{R}_{n}$.

And use (4.5) to obtain $|I| \leq 2 \tilde{R}_{n} \varepsilon$.

For II we first observe that,

$$
|\eta| \leq|\eta+\eta(t)|+|\eta(t)| \leq R_{0}(\varepsilon)+R_{n}=\tilde{R}_{n}
$$

and thus $\phi_{\tilde{R}_{n}}(\eta)=\eta$.

We rewrite $I I$ as

$$
\begin{aligned}
I I= & \int_{\mid \eta+\eta(t) \leq R_{0}(\varepsilon)}(\eta+\eta(t))|u(\eta, t)|^{2} \mathrm{~d} \eta \\
& -\eta(t) \int_{|\eta+\eta(t)| \leq R_{0}(\varepsilon)}|u(\eta, t)|^{2} \mathrm{~d} \eta \\
= & \int_{|\eta+\eta(t)| \leq R_{0}(\varepsilon)}(\eta+\eta(t))|u(\eta, t)|^{2} \mathrm{~d} \eta \\
& -\eta(t) M[u]+\eta(t) \int_{\mid \eta+\eta(t) \leq \leq R_{0}(\varepsilon)}|u(\eta, t)|^{2} \mathrm{~d} \eta \\
= & I I A+I I B+I I C
\end{aligned}
$$

Trivially, $|I I A| \leq R_{0}(\varepsilon) M[u]$, and by (4.5)

$$
|I I C| \leq|\eta(t)| \varepsilon \leq \tilde{R}_{n} \varepsilon .
$$

Thus,

$$
\begin{aligned}
\left|z_{\tilde{R}_{n}}^{\prime}(t)\right| & \geq|I I B|-|I|-|I I A|-|I I C| \\
& \geq|\eta(t)| M[u]-R_{0}(\varepsilon) M[u]-3 \tilde{R}_{n} \varepsilon
\end{aligned}
$$

Taking $t=\tilde{t}_{n}$, we can get

$$
\left|z_{\tilde{R}_{n}}^{\prime}\left(\tilde{t}_{n}\right)\right| \geq \tilde{R}_{n}(M[u]-3 \varepsilon)-R_{0}(\varepsilon) M[u]
$$

Combining (4.7), (4.8), and (4.9), we have

$$
\begin{aligned}
5 \varepsilon \tilde{t}_{n} \int_{0}^{\tilde{t}_{n}}\left|z_{\tilde{R}_{n}}^{\prime}(t)\right| \mathrm{d} t & \geq\left|\int_{0}^{\tilde{t}_{n}} z_{\tilde{R}_{n}}^{\prime}(t) \mathrm{d} t\right| \geq\left|z_{\tilde{R}_{n}}^{\prime}\left(\tilde{t}_{n}\right)-z_{\tilde{R}_{n}}^{\prime}(0)\right| \\
& \geq \tilde{R}_{n}(M[u]-5 \varepsilon)-2 R_{0}(\varepsilon) M[u] .
\end{aligned}
$$

Suppose $\varepsilon \leq \frac{1}{5} M[u]$ and use $\tilde{R}_{n} \geq R_{n}$ to obtain

$$
5 \varepsilon \geq \frac{\tilde{R}_{n}}{\tilde{t}_{n}}(M[u]-5 \varepsilon)-\frac{2 R_{0}(\varepsilon) M[u]}{\tilde{t}_{n}}
$$

Since $\frac{\tilde{R}_{n}}{\tilde{t}_{n}} \geq \varepsilon_{0}$ we have

$$
5 \varepsilon \geq \varepsilon_{0}(M[u]-5 \varepsilon)-\frac{2 R_{0}(\varepsilon) M[u]}{\tilde{t}_{n}}
$$

(Assume $\left.\varepsilon_{0} \leq 1\right)$ take $\varepsilon=\frac{M[u] \varepsilon_{0}}{16}$, as $n \rightarrow+\infty$, since $\tilde{t}_{n} \rightarrow+\infty$ we get a contradiction. 


\subsection{We Now Prove the Following Rigidity Theorem}

Lemma 4.2. If (1.5) and (1.6) hold, then for all $t$

$$
\|u(t)\|_{L^{2}}\|\nabla u(t)\|_{L^{2}}<\alpha\|\psi\|_{L^{2}}\|\nabla \psi\|_{L^{2}}
$$

where $\alpha=\left(\frac{M[u] E[u]}{M[\psi] E[\psi]}\right)^{\frac{1}{2}}$. We have also the bound for all $t$;

$$
\begin{aligned}
& 8\|\nabla u(t)\|^{2}-6\|u(t)\|_{L^{4}}^{4} \\
& \geq 8(1-\alpha)\|\nabla u(t)\|_{L^{2}}^{2} \geq 16(1-\alpha) E[u]
\end{aligned}
$$

The hypothesis here is $E[u]>0$ except if $u \equiv 0$. In fact, $E[u] \geq \frac{1}{6}\left\|\nabla u_{0}\right\|_{L^{2}}^{2}$.

Theorem 4.3. Assume $u_{0} \in H^{1}$ satisfies $P\left[u_{0}\right]=0$,

$$
M\left[u_{0}\right] E\left[u_{0}\right]<M[\psi] E[\psi]
$$

and

$$
\left\|u_{0}\right\|_{L^{2}}\left\|\nabla u_{0}\right\|_{L^{2}}<\|\psi\|_{L^{2}}\|\nabla \psi\|_{L^{2}} .
$$

Let $u$ be the global $H^{1}$ solution of (1.1) with initial data $u_{0}$ and assume that $K=\{u(\cdot-\eta(t), t) \mid t \in[0,+\infty)\}$ is precompact in $H^{1}$. Then $u_{0}=0$.

Proof. Let $\in C_{0}^{\infty}$ be redial with

$$
\varphi(\eta)=\left\{\begin{array}{ll}
|\eta|^{2} \text { for }|\eta| \leq 1 \\
0 & \text { for }|\eta| \geq 2
\end{array}\right\} .
$$

For $R>0$, we define

$$
z_{R}(t)=\int R^{2} \varphi\left(\frac{\eta}{R}\right)|u(\eta, t)|^{2} \mathrm{~d} \eta .
$$

Then

$$
z_{R}^{\prime}(t)=2 \operatorname{Im} \int R \nabla \varphi\left(\frac{\eta}{R}\right) \cdot \nabla u(t) \bar{u}(t) \mathrm{d} \eta .
$$

By the Hölder inequality:

$$
\begin{aligned}
\left|z_{\tilde{R}}^{\prime}(t)\right| & \leq c R \int_{|\eta| \leq 2 R}|\nabla u(t)||u(t)| \mathrm{d} \eta \\
& \leq c R\|\nabla u(t)\|_{L^{2}}\|u(t)\|_{L^{2}}
\end{aligned}
$$

By calculation, we have the local Virial identity

$$
\begin{aligned}
z_{R}^{\prime \prime}(t)= & 4 \sum_{j, k} \int \frac{\partial^{2} \varphi}{\partial \eta_{j} \partial \eta_{k}}\left(\frac{\eta}{R}\right) \frac{\partial u}{\partial \eta_{j}} \frac{\partial \bar{u}}{\partial \eta_{k}} \\
& -\frac{1}{R^{2}} \int\left(\Delta^{2} \varphi\right)\left(\frac{\eta}{R}\right)|u|^{2}-\int(\Delta \varphi)\left(\frac{\eta}{R}\right)|u|^{4} .
\end{aligned}
$$

Since $\varphi$ is radial we have

$$
z_{R}^{\prime \prime}(t)=\left(8 \int|\nabla u|^{2}-6 \int|u|^{4}\right)+A_{R}(u(t)) .
$$

where

$$
\begin{aligned}
A_{R}(u(t))= & 4 \sum_{j}\left(\int\left(\partial_{\eta_{j}}^{2} \varphi\right)\left(\frac{\eta}{R}\right)-2\right)\left|\partial \eta_{j} u\right|^{2} \\
& +4 \sum_{j \neq k} \int_{R \leq \eta \eta \mid \leq 2 R} \frac{\partial^{2} \varphi}{\partial \eta_{j} \partial \eta_{k}}\left(\frac{\eta}{R}\right) \frac{\partial u}{\partial \eta_{j}} \frac{\partial \bar{u}}{\partial \eta_{k}} \\
& -\frac{1}{R^{2}} \int\left(\Delta^{2} \varphi\right)\left(\frac{\eta}{R}\right)|u|^{2}-\int\left(\Delta \varphi\left(\frac{\eta}{R}\right)-6\right)|u|^{4} .
\end{aligned}
$$

Thus, we obtain

$$
\left|A_{R}(u(t))\right| \leq c \int_{|\eta| \geq R}\left(|\nabla u(t)|^{2}+\frac{1}{R^{2}}|u(t)|^{2}+|u(t)|^{4}\right) \mathrm{d} \eta
$$

Now discuss $z_{R}(t)$ for $R$ chosen appropriate large and selection time interval $\left[t_{0}, t_{1}\right]$ where $1 \ll t_{0} \ll t_{1}<\infty$. By (4.15) and (4.11) we have

$$
\left|z_{R}^{\prime \prime}(t)\right| \geq 16(1-\alpha) E[u]-\left|A_{R}(u(t))\right| .
$$

Set $\varepsilon=\frac{1-\alpha}{c} E[u]$ in (4.2), $R_{0} \geq 0$, such that $\forall t$

$$
\int_{|\eta+\eta(t)| \geq R}\left(|\nabla u|^{2}+|u|^{2}+|u|^{4}\right) \leq \frac{1-\alpha}{c} E[u] .
$$

Choosing $R \geq R_{0}+\sup _{t_{0} \leq t \leq t_{1}}|\eta|$. Then (4.16), (4.17) and (4.18) imply that for all $t_{0} \leq t \leq t_{1}$,

$$
\left|z_{R}^{\prime \prime}(t)\right| \geq 8(1-\alpha) E[u] .
$$

By Lemma 4.1, there exists $t_{0} \geq 0$ such that for all $t \geq t_{0}$ we have $|x(t)| \leq \mu t$ with $\mu>0$. By taking $R=R_{0}+\mu t_{1}$, we obtain that (4.18) holds for all $t_{0} \leq t \leq t_{1}$. Integrating (4.19) over $\left[t_{0}, t_{1}\right]$ we obtain

$$
\left|z_{R}^{\prime}\left(t_{1}\right)-z_{R}^{\prime}\left(t_{0}\right)\right| \geq 8(1-\alpha) E[u]\left(t_{1}-t_{0}\right) .
$$

On the other hand, for all $t_{0} \leq t \leq t_{1}$, by (4.10) and (4.14), we have

$$
\begin{aligned}
\left|z_{R}^{\prime}\left(t_{1}\right)\right| & \leq c R u(t)_{L^{2}}\|\nabla u(t)\|_{L^{2}} \leq c R\|\psi\|_{L^{2}}\|\nabla \psi\|_{L^{2}} \\
& \leq c\|\psi\|_{L^{2}}\|\nabla \psi\|_{L^{2}}\left(R_{0}+\mu t_{1}\right)
\end{aligned}
$$

Combining (4.20) and (4.21), we obtained

$$
8(1-\alpha) E[u]\left(t_{1}-t_{0}\right) \leq 2 c\|\psi\|_{L^{2}}\|\nabla \psi\|_{L^{2}}\left(R_{0}+\mu t_{1}\right) .
$$

It is important to mention that $\alpha$ and $R_{0}$ are constant depending only on $\frac{M[u] E[u]}{M[\psi] E[\psi]}$, and $t_{0}=t_{0}(\mu)$. Putting $\mu=\frac{(1-\alpha) E[u]}{c\|\psi\|_{L^{2}}\|\nabla \psi\|_{L^{2}}}$ and setting $t_{1} \rightarrow+\infty$, we 
obtain a contradiction except if $E[u]=0$, which implies $u \equiv 0$.

\section{REFERENCES}

[1] T. T. Tao, "On the Asymptotic Behavior of Large Radial Data for a Focusing Non-Linear Schrödinger Equation," Dynamics of Partial Differential Equations, Vol. 1, No. 1, 2004, pp. 1-48.

[2] T. Tao, "A (Concentration-)Compact Attractor for HighDimensional Non-Linear Schrödinger Equation," Dynamics of Partial Differential Equations, Vol. 4, No. 1, 2007, pp. 1-53.

[3] T. Cazenave, "Semilinear Schrödinger Equations. Courant Lecture Notes in Mathematics," New York University, Courant Institute of Mathematical Sciences, New York, American Mathematical Society, Providence, 2003.

[4] T. Tao, "Nonlinear Dispersive Equations: Local and Global Analysis," CBMS Regional Conference Series in Mathematics, Published for the Conference Board of the Mathematical Sciences, Washington DC, American Mathematical Society, Providence, 2006.

[5] M. Weinstein, "Nonlinear Schrödinger Equations and Sharp Interpolation Estimates," Communications in Mathematical Physics, Vol. 87, No. 4, 1982, pp. 567-576.

[6] J. Holmer and S. Roudenko, "A Sharp Condition for Scattering of the Radial 3D Cubic Nonlinear Schrödinger Equations," Communications in Mathematical Physics, Vol. 282, No. 2, 2008, pp. 435-467.

[7] C. E. Kenig and F. Merle, "Global Well-Posedness, Scattering, and Blow-Up for the Energy-Critical Focusing Nonlinear Schrödinger Equation in the Radial Case," Inventiones Mathematicae, Vo. 166, No. 3, 2006, pp. 645675.

[8] J. Colliander, M. Keel, G. Staffilani, H. Takaoka and T. Tao, "Global Existence and Scattering for Rough Solutions of a Nonlinear Schrödinger Equation on R3," Communications on Pure and Applied Mathematics, Vol. 57, No. 8, 2004, pp. 987-1014.
[9] T. Hmidi and S. Keraani, "Blowup Theory for the Critical Nonlinear Schrödinger Equations Revisited," International Mathematics Research Notices, Vol. 2005, No. 46, 2005, pp. 2815-2828. doi:10.1155/IMRN.2005.2815

[10] F. Merle and Y. Tsutsumi, " $\mathrm{L}^{2}$ Concentration of Blow-Up Solutions for the Nonlinear Schrödinger Equation with Critical Power Nonlinearity," Journal of Differential Equations, Vol. 84, No. 2, 1990, pp. 205-214.

[11] W. A. Strauss, "Existence of Solitary Waves in Higher Dimensions," Communications in Mathematical Physics, Vol. 55, No. 2, 1977, pp. 149-162.

[12] D. Foschi, "Inhomogeneous Strichartz Estimates," J. Hyper. Diff. Eq., Vol. 2, No. 1, 2005, pp. 1-24.

[13] C. Kenig, G. Ponce and L. Vega, "Well-Posedness and Scattering Results for the Generalized Korteweg-De Vries Equation via the Contraction Principle," Communications on Pure and Applied Mathematics, Vol. 46, No. 4, 1993, pp. 527-620.

[14] S. Keraani, "On the Defect of Compactness for the Strichartz Estimates of the Schrödinger Equation," Journal of Differential Equations, Vol. 175, No. 2, 2001, pp. 353392. doi:10.1006/jdeq.2000.3951

[15] E. Donley, N. Claussen, S. Cornish, J. Roberts, E. Cornell and C. Wieman, "Dynamics of Collapsing and Exploding Bose-Einstein Condensates," Nature, Vol. 412, No. 6844, 2001, pp. 295-299. doi: $10.1038 / 35085500$

[16] C. Sulem and P.-L. Sulem, "The Nonlinear Schrödinger Equation. Self-Focusing and Wave Collapse," Applied Mathematical Sciences, Vol. 139, 1999.

[17] M. Vilela, "Regularity of Solutions to the Free Schrödinger Equation with Radial Initial Data," Illinois Journal of Mathematics, Vol. 45, No. 2, 2001, pp. 361-370.

[18] L. Bergé, T. Alexander and Y. Kivshar, "Stability Criterion for Attractive Bose-Einstein Condensates," Physical Review A, Vol. 62, No. 2, 2000, 6 p.

[19] P. Bégout, "Necessary Conditions and Sufficient Conditions for Global Existence in the Nonlinear Schrödinger Equation," Advances in Applied Mathematics and Mechanics, Vol. 12, No. 2, 2002, pp. 817-827. 